\title{
Review Paper on Implementation of Particle Swarm Optimization for Multi-Pass Milling Operation
}

\author{
Sagar Bahirje, Dr.Vishvajeet Potdar, \\ A. G.Patil Institute of Technology Solapur, \\ Punyashlok Ahilyadevi Holkar Solapur University \\ Maharashtra-413006 India
}

\begin{abstract}
Now a day's optimizing machining process parameters, various effective techniques such as Particle Swarm Optimization (PSO), Genetic Algorithm (GA), Simulated Annealing (SA), Ant Colony Optimization (ACO) and Artificial Bee Colony algorithm (ABC) have been used. In This paper we give an overview of PSO techniques to optimize milling machining process parameter of both traditional and modern machining from 2011 to 2018. Machining process parameters such as cutting speed, depth of cut and tool condition is mostly considered by researchers in order to minimize or maximize machining performances. From the review, the most machining process considered in PSO was multi-pass turning while the most considered machining performance was production costs.
\end{abstract}

\section{INTRODUCTION}

The main criterion in process planning of any machining operation is to select the appropriate machining parameters. Generally, the machining parameters are decided based on the literature or using any machining data hand book. But it is a well-known reality that the process parameters selected from these resources are far away from the optimal process parameters. The other method to choose the process parameters is by performing trials of experiments. But the trials made always may not be accurate and they may lead to some error. Hence carrying out such trials may not be ultimate solution as cost and time factors are involved. Nowadays, in manufacturing environment the computer numerical control (CNC) machines need to be utilized to the fullest to get back the valuable pay back.

The economic utilization of the CNC machines is mainly dependent on the appropriate selection of the machining parameters, as well as reduction of the machining time. In the present scenario, development of new generation of reinforced materials provides considerable tailor ability of mechanical properties. Among several types of MMCs, aluminum matrix composites (AMMCs) have gained greater exposure in automobile and other industries due to light weight, high strength-to stiffness ratio, alterable thermal and electrical properties, etc. the properties of tool material. Investigation of process parameters on surface integrity of machining of AMMCs using coated inserts has not been addressed. Therefore, effect of process parameters on surface integrity is analyzed using graphical representation.

It is necessary to develop a technique of predicting the significant process parameters before actual machining is carried out to obtain the desired responses. Generally, the non-traditional optimization techniques are incorporated to overcome the aforesaid issues. In the present study, the PSO is carried out and compared with Desirability approach to identify the machining parameters to obtain the desired responses.

\section{LITERATURE REVIEW}

W.A. Khan [1] the effect of acceptance probability and node perturbation scheme on convergence has been monitored for varying problem size and topology. It is determined that for a specific problem size, the iteration required to achieve a globally optimal solution is dependent upon the number of nodes and their distribution. In real industrial situation, the problem instances shall have variable size and unpredictable node distribution. Simulated annealing algorithm gives optimal solution. A sub-optimal solution to justify the computing cost may be obtained at reduced iterations. It is also possible to use other TSP solution algorithms for the solution of this problem situation. Necessary modification shall however be required to introduce approach and exit constraints.

Anurag Sharma [2] In this paper, they have presented for the first time a hybrid PSO-GMDH framework because we are not aware of any literature that has reported this. Two types of problems have been solved, one is based on experimentation in a manu-facturing laboratory in which the controlling parameters were used to generate output response in the form of tool wear; the other is the time series problem. Although the results obtained are not as competitive as those obtained in chapter 4 for tool wear problem, they are quite promising.

The man-machine interface for the hybrid PSO-GMDH software developed and reported in this chapter is very conducive for utilizing as a data mining platform.

Pinkey Chauhan [3] the present study discusses the problem of process parameter optimization during turning operations. The multi-pass operations are dominantly preferred over single pass operations from economic standpoint. The selection of optimal machining parameters such as cutting speed, feed rate, depth of cut during rough and finish passes drastically affects the quality and production cost of the machined part. Therefore process parameter optimization is desired to serve the consumer production demands in terms of good quality product with 
lower costs. A new chaotic PSO, namely Totally Disturbed PSO (TDPSO), is used for optimization and its performance is analyzed by comparing results with previous studies. Two empirical machining models simulating multi-pass turning process are considered and evaluated for optimal machining conditions. Since the machining operations are performed under a constrictive machining environment therefore both the machining models are highly constrained and nonlinear in nature. The objective is to determine those values of process parameters which minimize the total production cost. The numerical results show the competence of TDPSO for dealing machining parameter optimization of multi-pass operations. It may be concluded that TDPSO is a good choice for solving complex machining optimization problems arising in manufacturing or other process industries.

Yang Yang [4] this paper proposes a novel nonlinear constrained optimization for multi-pass milling operations, which combines ICA and constraints handling strategies. The effectiveness of algorithm has been demonstrated by an application case compared with previous work. The results reveal that a new and effective method has been proposed for the optimization of machining parameters in multi-pass milling process.

Rashmi Laxmikant Malghan [5] The study was carried in two stages. In the initial stage, the trial experiments were conducted to identify the appropriate ranges of the process parameters. In the next stage, the interaction between the process parameters and the responses was determined by adopting the regression equations. Finally, the multiobjective optimization was performed using the desirability approach and PSO technique. The following findings were drawn from the study:

1. From the ANOVA results, it was found that spindle speed has major contribution followed by feed rate and depth of cut on all the responses.

2. To attain better $\mathrm{Ra}$ for the aforesaid material, it is necessary to apply higher spindle speed, higher depth of cut and lower feed rate. Feed rate has prominent effect on $\mathrm{Ra}$ as compared to spindle speed and depth of cut.

3 . The predictability of the multiple regression approach is found to be $99.89 \%$ for $\mathrm{FX}, 98.88 \%$ for $\mathrm{Ra}$ and for power consumption. $99.83 \%$.

4. The outcomes gained through PSO are compared with the desirability approach and it was observed that PSO gives more accurate values in contrast to the results attained with the desirability approach.

5. The results of confirmation experiments show the effectiveness of PSO in predicting the desired responses such as FX, Ra and power consumption.

Norfadzlan Yusup [6] as they can be summarized that the most machining processes considered in PSO was Multipass-turning followed by end milling and milling machining. For machining performance, the most machining performance measured was machining/production costs followed by surface roughness and machining/production time. From the various researches in the literature, it was proven that PSO performed better than other soft computing techniques such as GA and SA. PSO was employed by most researchers because of the simplicity and in addition it also has a features of both GA and evolution strategies.

N. Baskar [7] In this work, different non-conventional optimization techniques such as GA, CACO, TS and PSO algorithm have been studied for the optimization of machining parameters in milling operations.

Optimization procedures based on the above approaches have been developed and are successfully implemented. Significant improvement is obtained with the above techniques in comparison to the results by handbook and method of feasible direction.

This is because of the limitations of enumerative search followed by the direct search method. In the use of nonconventional techniques, PSO always yields better results. So it is suggested to use PSO for solving the optimization problem of the milling operations. Results obtained in this work are intended for use by NC machines. However they can also be used for manually operated machines. It is also observed that the procedures described in this work can be applied to similar machining operations as well as other nonlinear optimization problems.

N. Baskar [8] Economy of the machining operations has been the worry of most of the manufacturing firms, as they invest a lot in purchasing the machines, especially the CNC machines. As it is well known that optimization of machining parameters matter a lot in the economy of machining operations, a lot of search are being carried out to achieve the above said for various operations. So far, the focus was only towards the turning operations and only a little attention has been paid to other important operations like, milling, grinding, drilling, etc. In this work, a specific case in milling operation was taken and solved by using three different non-traditional optimization techniques which comprises of:

1. Global search algorithm (genetic algorithm).

2. Local search algorithm (local hill climbing).

3. Memetic algorithm.

Optimization procedures based on the above approaches have been developed and are successfully implemented. Significant improvement is obtained with the above techniques in comparison with the results by handbook and method of feasible direction. This is because of the limitations of enumerative search followed by the direct search method. In the use of non-conventional techniques, memetic algorithm always yields better results. So it is suggested to use memetic algorithm for solving the optimization problem of the milling operations. Results obtained in this work are intended for use by NC machines. However they also be used for manually operated machines. It is also observed that the procedures described in this work can be applied to similar machining operations as well as other non-linear optimization problems.

Devarasiddappa Devarajaiah [9] In this paper, fuzzy logicbased model is developed for surface roughness prediction during end milling of Al-SiCp metal-matrix composites.

- Predictive capability of the developed fuzzy rule-based surface roughness model is verified with experimental work and obtained mean error as $4.56 \%$ while mean squared error is recorded as 0.073 . 
- ANOVA performed at $95 \%$ confidence level showed that feed rate $(69.93 \%)$ bears significant statistical influence on surface roughness followed by SiCp content $(19.71 \%)$ and spindle speed $(9.73 \%)$.

Surface roughness value varies directly as the feed rate and percentage of $\mathrm{SiCp}$ in the metal-matrix composite and varies inversely with the spindle speed.

- The interaction between SiCp percentage and feed rate has significant effect compared to other interaction factors on surface roughness during machining of Al-SiCp metalmatrix composite. Lower feed rate with higher spindle speed produces good surface finish during end milling $\mathrm{Al} / \mathrm{SiCp}$ metal-matrix composite using carbide tool.

P. Asokan [10] In this work, different non-conventional optimization techniques such as GA, CACO, TS and PSO algorithm have been studied for the optimization of machining parameters in milling operations.

Optimization procedures based on the above approaches have been developed and are successfully implemented. Significant improvement is obtained with the above techniques in comparison to the results by handbook and method of feasible direction.

This is because of the limitations of enumerative search followed by the direct search method. In the use of nonconventional techniques, PSO always yields better results. So it is suggested to use PSO for solving the optimization problem of the milling operations. Results obtained in this work are intended for use by $\mathrm{NC}$ machines. However they can also be used for manually operated machines. It is also observed that the procedures described in this work can be applied to similar machining operations as well as other nonlinear optimization problems.

\section{CONSTRAINTS FOR OPTIMIZATION}

In practice, possible range of cutting speed and feed rate are limited by the following constraints

1. Maximum machine power

2. Surface finish requirement

3. Maximum cutting force permitted by the rigidity of the tool

4. Available feed rate and spindle speed on the machine tool.

\section{CONCLUSION}

As we study a many number of paper, we can be summarized that the most machining processes considered in PSO was Multipass -turning followed by end milling and milling machining. For machining performance, the most machining performance measured was machining/production costs followed by surface roughness and machining/production time. From the various researches in the literature, it was proven that PSO performed better than other soft computing techniques such as GA and SA. PSO was employed by most researchers because of the simplicity and in addition it also has a features of both GA and evolution strategies.

\section{REFERENCE}

[1] W.A. Khan a, D.R. Hayhurst b, C. Cannings, 1999, Determination of optimal path under approach and exit constraints. European Journal of Operational Research 117 (1999) $310 \pm 325$

[2] Anurag Sharma and Godfrey Onwubolu, Hybrid Particle Swarm Optimization and GMDH System.

[3] Pinkey Chauhan - Millie Pant - Kusum Deep Parameter optimization of multi-pass turning using chaotic PSO, SpringerVerlag Berlin Heidelberg 2014.

[4] Yang Yang, Xinyu Li, Liang Gao, Parameters Optimization of a Multi-pass Milling Process Based on Imperialist Competitive Algorithm, Proceedings of the 2013 IEEE 17th International Conference on Computer Supported Cooperative Work in Design.

[5] Rashmi Laxmikant Malghan, Karthik M. C. Rao, Arun Kumar Shettigar, Application of particle swarm optimization and response surface methodology for machining parameters optimization of aluminium matrix composites in milling operation, The Brazilian Society of Mechanical Sciences and Engineering 2016.

[6] Norfadzlan Yusup, Azlan Mohd Zain, Siti Zaiton Mohd Hashim, Overview of PSO for Optimizing Process Parameters of Machining, International Workshop on Information and Electronics Engineering (IWIEE).

[7] N. Baskar . P. Asokan . R. Saravanan .G. Prabhaharan, Optimization of Machining Parameters for Milling Operations Using Non-conventional Methods, Springer-Verlag London Limited 2004. 